\title{
Design of Measuring System with Metamaterial Inspired CSRR
}

\author{
Rucha R. Sarwadnya ${ }^{1}$, Prof. N. A. Dawande ${ }^{2}$ \\ P.G. Student, Department of Electronics and Telecommunication Engineering, Dr. D. Y. Patil College of Engineering, \\ Ambi, Pune, Maharashtra, India ${ }^{1}$ \\ Associate Professor, Department of Electronics and Telecommunication Engineering, Dr. D. Y. Patil College of \\ Engineering, Ambi, Pune, Maharashtra, India ${ }^{2}$
}

\begin{abstract}
This paper presents a design of measuring system with Metamaterial inspired CSRR (Complimentary SplitRing Resonator). The device is of portable, cost effective and which gives the accuracy. The theoretical approach of Metamaterial with its different applications is also discussed here. This paper is of high significance as current testing equipment of micro Complimentary Split-Ring Resonators (CSRR) are highly expensive, and therefore rare, but are also extremely bulky. Therefore developing portable, cost-effective, and robust testing equipment operating within the internationally reserved ISM radio frequency band is a main research work.
\end{abstract}

Keywords: Complimentary Split-Ring Resonators (CSRR), Metamaterial, Sensor, Permeability.

\section{INTRODUCTION}

We are aware of sensor device that detects and responds to some type of input from the physical environment. The specific input could be light, heat, motion, moisture, pressure, or any one of a great number of other environmental phenomena. The output is generally a signal that is converted to human-readable display at the sensor location or transmitted electronically over a network for reading or further processing.

Recently, significant advances have been done for material characterization using properties techniques. Some of these techniques are presented for the real part of the dielectric constant measurement; conversely, each technique is suitable and valuable for certain kinds of frequency ranges and certain kinds of materials. Material characterization has an application in various areas such as bio-sensing, quality control in the food industry, substrate properties and so on [1]. In modern years, Metamaterial became one of the main topics of current research in the field of high-frequency technology. Metamaterial are a combination of conductive and non-conductive materials, whose arrangement and characteristics lead to new and exotic properties that do not occur in nature. These materials can show a negative permeability and permittivity in certain frequency ranges, whereby a negative refractive index is possible [2].

\section{NOVEL CSRR SENSORS}

These exhibit extremely unique set of properties, which are not observed in nature like negative permittivity or negative permeability or both simultaneously, at any desired frequency from radio waves to optical depending on structural extent and arrangement. The arrangements in such structures are more important than the constituents of the material, which finds unique applications as: Super lens, Cloaks, Stealth, Antennas, Absorbers, Phase compensator, Sensors [3]. Cheap and ubiquitous sensor systems will shape the coming decades. There is an emerging class of tiny high performance electronic devices such as mobile phones, electronic toys, home appliances, monitoring and control systems in industrial amenities, and medical diagnosis systems, which are or will be equipped with pill box sized microprocessors, computers as well as sensors. These "smart sensors" with limited power and processing capabilities are frequently wirelessly interconnected. An assembly of many of them spread throughout the physical world will form sensor networks able to recognize, localize, and monitor physical, environmental, and industrial processes, biological and health situation, goods, vehicles, factories, stores, or even people [4].

\section{III.METAMATERIAL BASED DEVICES}

Man-made meta-atoms: Natural materials consist of large quantities of fundamental particles, atoms or molecules. The macroscopic electromagnetic (EM) properties of a material are usually presented by the electric permittivity and the magnetic permeability.

In contrast, metamaterial are composed of periodically or non-periodically arranged unit cells of man-made metaatoms. These meta-atoms are essentially sub-wavelength electric or/and magnetic resonators which response to the external electromagnetic fields as harmonic oscillators [5].

The followings are some applications of metamaterial. 


\section{A. Antennas:}

Metamaterial antennas are a class of antennas which use Metamaterial to increase performance of miniaturized (electrically small) antenna systems. For example, a wideband horn antenna (loaded with metamaterial-inspired particles) can be enabled to perform different manipulations on the transmitted and received signals, such as band-pass and band-stop filtering processes, and polarization transformation [5].

\section{B. Absorbers:}

A Metamaterial absorber is intended to efficiently absorb electromagnetic radiation such as light. structure is designed to broaden the bandwidth of metamaterial absorber composed of a patterned square metallic patch and a metallic ground plane separated by a dielectric layer [6]. The conductivity of the metal and the thickness of the dielectric layer determine the bandwidth of the metamaterial absorber [7].

\section{Super lens:}

A Super lens is a lens which uses Metamaterial to go beyond the diffraction limit. The diffraction limit is a feature of conventional lenses and microscopes that limits the fineness of their resolution. A two-dimensional Luneburg lens based on bulk Metamaterial is composed by a number of concentric layers [8].

\section{Cloaks:}

Metamaterial cloaking is the usage of Metamaterial in an invisibility cloak. A new pi-shaped metamaterial unit cell that exhibits more than a $2 \mathrm{GHz}$ wideband near zero refractive index (NZRI) property in the microwave region. The material was then utilized in the design of three types of cloak shapes (rectangular-shaped, eye-shaped, and triangular shaped) for hiding a metal cylinder. It was found that, for all the three types of shapes, the metal object could be cloaked in the $\mathrm{C}$-band region by reducing the normalized scattering width to below zero, where the material was showing near zero refractive index property [9].

\section{E. Sensors:}

There are varieties of metamaterial sensors which can be used for specific application. These sensors are Biosensor (microwave, terahertz, plasmonic), Thin-film sensor, Wireless strain sensor, etc [10]. A microwave sensor based on an artificial transmission line is used for non-invasive blood glucose monitoring. Corresponding numerical model shows dimensions of the sensor leading to sensitivity suitable for accurate monitoring of glucose in blood [11].

\section{IV.MICRO STRIP BASED METAMATERIAL CSRR}

These CSRR structures, in general, are dual complements of SRRs which exhibit negative permittivity by electric field excitation. Since CSRRs are excited by electric field perpendicular to its plane, they show more sensitivity to

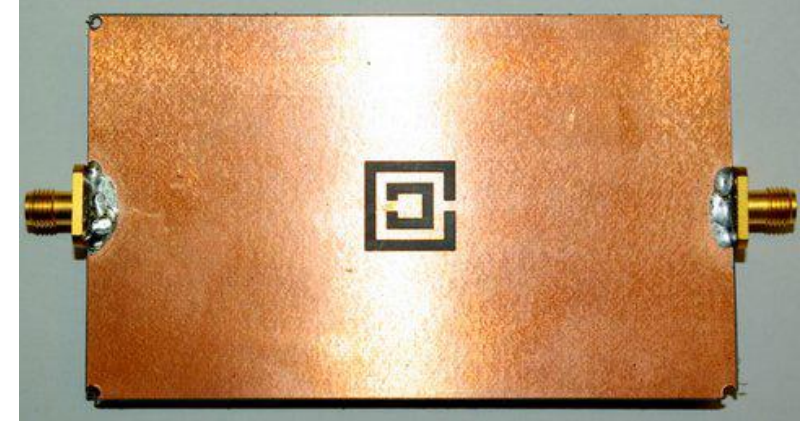

Fig.1 Micro strip-based Metamaterial CSRR Sensor

change in permittivity of the surroundings and have better sensitivity than SRRs. CSRRs till now have been explored for dielectric characterization of planar materials, antenna, and filters. In short, the sensor which is proposed is low cost, simple design, easy to fabricate, extremely economical, sensitive, selective, and ultra-fast sensingcum-recovery sensor for hybrid fuel sensing. Furthermore, two different liquids (all liquids used in this study depict $100 \%$ concentrations) have been sensed using the CSRRs to use them for hybrid fuel sensing, namely, petrol and ethanol. Working at $4.5 \mathrm{GHz}$ resonant frequency, this design was optimized based on the distribution of electrical field to measure the permittivity. The results were simulated using CST Microwave Studio software and further verified experimentally. The response was extremely fast (comparable to data acquisition time) and the same was true for recovery time [12].

\section{PROPOSED WORK}

This proposed work aims to develop a highly accurate, cost effective, and portable prototype measuring system capable of accurately testing the sensitivity of a range of Metamaterial-based micro chemical sensors. The prototype measuring equipment will consist of two components. First, a Frequency Generator which, in a controlled way, can input selected micro-wave frequencies that reflect the chemical characteristics of specific fluids. The second component is a Frequency Detector which measures the RMS value of the sensor's radio frequency. Therefore developing and making available cost-effective and robust testing equipment capable of effectively measuring the accuracy of these CSRRs will represent a significant breakthrough in enabling the wider use and applications of CSRR sensors [13].

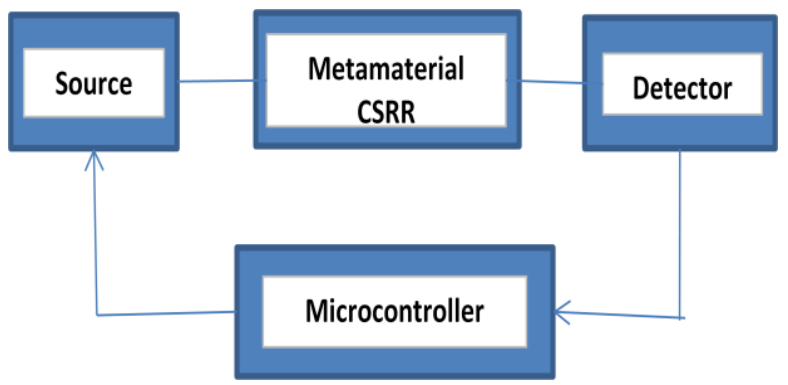

Fig.2 Proposed System Block Diagram 


\section{VI.CONCLUSION}

The Metamaterial inspired complimentary split ring resonator based proposed system will overcome the limitations of the current generation of high cost, low availability and bulky measuring and sensing devices by developing a prototype that not only provides high levels of accuracy at a wide range of temperatures but is low in cost and is highly portable. Also it will introduce to a new concept of metamaterial inspired micro strip sensor application for hybrid fuel detection.

\section{REFERENCES}

[1] Rammah, A.A., Zakaria, Z., Ruslan, E., Isa, A.A.M. (2015). Comparative study of materials characterization using microwave resonators. Australian Journal of Basic and Applied Sciences, 9, 7685.

[2] Von Detten, F., Basten, D., And Ajami, A., "Low Cost Proximity Sensor System Using Metamaterial Structures," Conference paper, January 2013

[3] M. Saif Islam and Logeeswaran VJ, University of California, Davis, "Nanoscale Materials and Devices for

[4] Future Communication Networks," IEEE Communication Magazine, June 2010.

[5] Schüßler, M., Mandel, C., Puentes, M. \& Jakoby, R."Metamaterial Inspired Microwave Sensors" IEEE Microwave Magazine, March/April 2012.

[6] Mirko Barbuto, Davide Ramaccia, Fabrizio Trotta, Filiberto Bilotti, and Alessandro Toscano, "Signal manipulation through horn antennas loaded with metamaterial-inspired particles: A review," EPJ Applied Metamaterials, 2015.

[7] Soni P. , and Meena S.," A Review paper on Metamaterial Absorber," International Journal of Scientific \& Engineering Research, Volume 6, Issue 10, October 2015.

[8] B.X.Wang ,L.L.Wang , G.Z.Wang , W.Q.Huang , X.Zhai , and X.F.Li ,'Tunable Bandwidth of Terahertz Metamaterial Absorber," Optical Communication Journal,vol.325, June.2014.

[9] Haibing Chen, Qiang Cheng, Aihua Huang, Junyan Dai, Huiying Lu, Jie Zhao, Huifeng Ma, Weixiang Jiang, and Tiejun Cui, "Modified Luneburg Lens Based on Metamaterials," International Journal of Antennas and Propagation, Article ID 902634, 2015.

[10] Sikder Sunbeam Islam, Mohammad Rashed Iqbal Faruque and Mohammad Tariqul Islam, " A Near Zero Refractive Index Metamaterial for Electromagnetic Invisibility Cloaking Operation," Materials, July 2015.

[11] WenXuan Tang, ZhongLei Mei, and TieJun Cui, "Theory, experiment and applications of metamaterials," Springer, Science China, December 2015.

[12] Jan VRBA and David VRBA, "A Microwave Metamaterial Inspired Sensor for Non-Invasive Blood Glucose Monitoring," RADIOENGINEERING, Vol. 24, No. 4, December 2015.

[13] Kaushal Gangwar, Dr.Paras and Dr. R.P.S Gangwar, "Metamaterials: Characteristics, Process and Applications," Advance in Electronic and Electric Engineering. ISSN 2231-1297, Volume 4, Number 1, 2014, pp.97-106.

[14] Rawat, V., Dhobale, S., \& Kale, S. "Ultra-fast selective sensing of ethanol and petrol using microwave-rangemetamaterial complementary split-ring resonators," Journal of Applied Physics, 116, 164106, 2014. 